\title{
Human-preference-based Control Design: Adaptive Robot Admittance Control for Physical Human-Robot Interaction
}

\author{
Vladislav Okunev, Thomas Nierhoff, Sandra Hirche
}

\begin{abstract}
Aiming at the application in physical human-robot interaction, this paper presents a novel adaptive admittance control scheme for robotic manipulators. Special emphasis is drawn on the avoidance of oscillatory behavior in the presence of closed kinematic chains while keeping the rendered impedance low. The approach uses an online fast Fourier transform of the measured manipulator endeffector forces in order to detect oscillations and to adapt the admittance parameters dynamically. As a novel method towards humancentered control design the adaptation strategy is determined in a user study evaluated with a machine-learning algorithm. Experiments conducted with ten human participants show superiority over the non-adaptive admittance control scheme.
\end{abstract}

\section{INTRODUCTION}

Anthropomorphic robots designed to interact with humans in unknown environments are an active field of research. Unlike industrial robots, those robots are designed to operate in close contact with humans. A common approach to minimize internal and unintended collision forces during the interaction are compliant control strategies like the admittance control. Still, the control design for interaction with rigid environments, closed kinematic chains, or cooperative tasks involving humans is challenging. In these situations, constant admittance parameters are not suitable.

Various adaptive admittance control approaches are proposed in literature in order to improve the interaction of a robotic manipulator with its environment. Focusing on stiffness adjustment, the goal presented in [1] is to ensure a constant interaction force during contact with the environment. In [2], an adaptive controller is developed which varies the desired admittance depending on the robot's position tracking error. Shown in [3] is an adaptive approach which uses a variation of the admittance parameters to react dynamically to environmental impedance changes by adjusting the robot's viscosity coefficient dynamically. Unlike software-based control strategies, there also exist hardware based approaches like [4]-[6], where mechanical construction elements are used to achieve variable link compliance.

Other approaches focusing on adaptive admittance control consider a task-dependent human centered admittance adjustment in order to improve the robot's performance. In [7], the human stiffness characteristics in a cooperative task between two humans are analyzed. Based on the obtained results a switching strategy between two human-like fixed

Part of this work is the result of Vladislav Okunev's bachelor thesis. Vladislav Okunev, Thomas Nierhoff and Sandra Hirche are with the Institute of Automatic Control Engineering (LSR), Faculty of Electrical Engineering, Technische Universität München, D-80290 München, Germany v.okunev@mytum.de, \{tn, hirche\}@tum.de admittance parameter sets is presented. The same authors derive an optimal solution for computing the damping factor by minimizing a cost function in [8]. In [9], a human-like admittance parameter adaptation scheme for service tasks is combined with an adaptation strategy depending on the type of human-robot interaction. Similarly, the works presented in [10], [11] rely on situation-triggered parameter adaptation. In [10] the admittance parameter adaptation is based on computing the angle between force and velocity vector, thus making it possible to reason about the current interaction scenario. Differentiation of the force as a natural measure of the human intention is proposed in [11], allowing one to modify the impedance parameters accordingly.

Summing up, existing literature focuses mainly on the task-dependent admittance parameters adaptation, while the human-specific preferences are disregarded. Little consideration is given to the development of suitable admittance control schemes tackling the problem of resonance oscillations as particularly induced by closed kinematic chains. Especially in tightly-coupled tasks involving one or more robot manipulators and an interacting human, resonance oscillations may occur for certain admittance control parameters, resulting in an unsuccessful task completion.

The contribution of this paper is the development of a variable admittance control strategy that is able to adapt dynamically to environmental changes in order to avoid oscillative behaviour of the robotic manipulator. The adaptation strategy is designed to improve the haptic interaction experience of the cooperating human partner by choosing appropriate admittance parameters. For this purpose a novel method to derive the adaptation strategy in a human user study evaluated by a machine-learning algorithm is proposed. More specifically, a trained frequency-domain classifier using AdaBoost is considered. The oscillations are detected online based on measured endeffector forces and canceled through appropriate parameter adaptation. Evaluation of the entire system is performed in a human user study with ten participants demonstrating the superiority of the resulting adaptive control scheme over a non-adaptive counterpart.

The remainder of this paper is organized as follows: Sec. II introduces the problem statement, which is tackled in detail in Sec. III through a suitable adaptation scheme. In Sec. IV the performance of the overall system is evaluated through human user studies. Sec. V critically discusses the proposed algorithm with regard to its potential and limitations. A conclusion and proposals for further enhancement can be found in Sec. VI. 


\section{PRoblem STATEMENT AND GENERAL APPROACH}

The example scenario considered in this paper consists of an cooperative task between a human and two manipulators holding a rigid object together.

In general, it is desired to keep the rendered inertia and damping values of robotic manipulators in interactive tasks as low as possible. However, in case of too small values, admittance-controlled manipulators tend to oscillate at their resonance frequencies depending on the set of admittance parameters chosen, the type of coupling, the type of interaction, and the natural dynamics of the manipulator. While some frequencies may disturb the haptic experience of the interacting human partner, others may not even be perceivable. Therefore, the goal is to design the admittance control scheme such that:

- inertia and damping are kept as low as possible

- oscillations disturbing the haptic experience are suppressed.

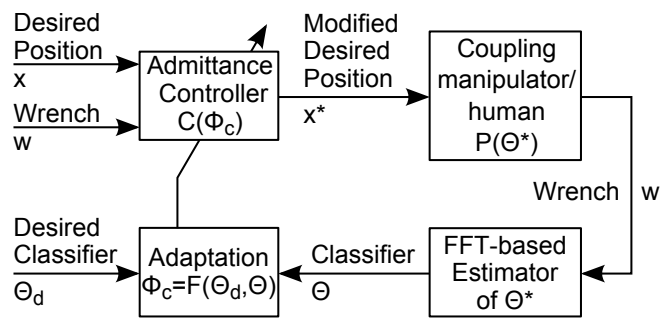

Fig. 1. Adaptive control scheme

We employ a control approach which is inspired by the direct adaptive control scheme [12] and depicted in Fig. 1: Given a desired position $\mathbf{x} \in \mathbb{R}^{6}$ in task space and a measured wrench $\mathbf{w} \in \mathbb{R}^{6}$, the admittance control scheme of each manipulator creates an adequate modified desired position $\mathbf{x}^{*}$. During interaction, the human's subjective perception of the oscillation intensity is described by a parameter $\Theta^{*} \in \mathbb{R}$. In order to approximate the parameter $\Theta^{*}$ as good as possible and derive an optimized control scheme, human user studies in combination with a boosting-based learning process for the oscillatory behavior recognition are performed. During runtime the parameter $\Theta^{*}$ is estimated online using an FFT in combination with the obtained boosted classifiers. The result of the classification process is denoted $\Theta \in \mathbb{R}$. By comparing $\Theta$ and a desired classifier $\Theta_{d} \in \mathbb{R}$ representing the optimal interaction experience for the human, a suitable adaptation scheme for the admittance control inertia, damping and spring parameters $\Phi_{c}=\{\mathbf{M}, \mathbf{D}, \mathbf{K}\}$ is found.

\section{AdAptive Admittance Control}

This section covers the approach of developing an adaptive admittance control scheme fulfilling the requirements presented in Sec. II. It is first analyzed which admittance control parameters are suitable for adaptation. Next, the FFT-based boosting process is presented allowing one to develop a suitable classifier offline considering specific user

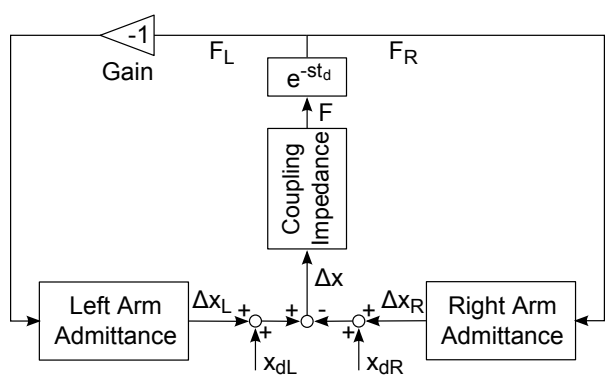

Fig. 2. Model of two coupled admittance-controlled manipulators used for numerical oscillation evaluation.

preferences. Finally, an adaptive control scheme is proposed based on the obtained results.

\section{A. Adaptation parameter selection}

Given the scheme in Fig. 1, the influence of varied admittance parameters and the selection of a suitable set of adaptive parameters $\Phi_{c}$ is covered in this section. For this purpose, a model of two coupled manipulators as displayed in Fig. 2 is used. The model consists of two blocks for the admittance control loops of the left and right robot manipulator (called arm) described by

$$
\Delta x_{R / L}=\left(M_{a} s^{2}+D_{a} s+K_{a}\right)^{-1} F_{R / L} .
$$

For reasons of simplicity the blocks are equally parameterized with arm inertia $M_{a}$, arm damping $D_{a}$ and arm spring constant $K_{a}$. The variables $\Delta x_{R}$ and $\Delta x_{L}$ denote the resulting positional offset for the right respectively left arm depending on the applied external force $F_{R}$ and $F_{L}$. The variable $s$ stands for the Laplace transform variable. In case both arms are coupled through an object being grasped, the coupling is modeled as a spring-mass-damper system connecting the endeffectors according to

$$
F=\left(M_{i} s^{2}+D_{i} s+K_{i}\right) \Delta x
$$

with coupling mass $M_{i}$, coupling damping $D_{i}$ and coupling spring constant $K_{i}, F$ as the coupling force and $\Delta x$ as the positional offset between the left and the right arm. The coupling spring constant determines the stiffness of the coupling link. Any delay or time constant in the overall model is represented with a concentrated delay $t_{d}$ according to

$$
F_{R}=-F_{L}=F \cdot e^{-s t_{d}},
$$

where the inverse relation between the external forces acting at the left and the right endeffectors is caused by the coupling.

Implicitly, the following simplifications are made:

- The underlying position controller of each arm is high gain, i.e. the position error is negligible.

- The human impedance is included in the coupling impedance and represented by a suitable set of coupling parameters $M_{i}, D_{i}$ and $K_{i}$.

- Only one translational dimension is considered. Thus the wrench $\mathbf{w}$ in Fig. 1 becomes a force $F$ in Fig. 2. 
- All delays and time constants due to delays in the control loop, a finite sampling time, filtering of the wrench values $F_{R}$ and $F_{L}$, mechanical and electrical time constants are encoded in $t_{d}$.

The effect of varied parameters is evaluated through numerical simulations using a bisection algorithm and shown in Fig. 3. Simulation results are obtained through running the simulation for $100 \mathrm{~s}$ after displacing both arms $0.5 \mathrm{~m}$ from their desired position to excite all frequencies. The system is said to be oscillatory whenever $\left\{\left|\Delta x_{R}\right|,\left|\Delta x_{L}\right|\right\}>1$ during the simulation or $\Delta x_{R}$ or $\Delta x_{L}$ do not converge to a fixed position at the end of the simulation. Shown on the left side of Fig. 3 is the effect of a varied arm inertia and damping depending on the overall time delay $t_{d}$. Increasing the arm inertia $M_{a}$ leads to a less oscillatory effect whereas increasing the arm damping $D_{a}$ only helps reducing scillations for $D_{a}<2 \cdot 10^{3} \frac{\mathrm{Ns}}{\mathrm{m}}$. The right side displays the effect of a varied coupling inertia $M_{i}$ and damping $D_{i}$. With a given admittance parameterization of $M_{a}, D_{a}$, there exists an upper boundary for the coupling impedance parameters which should not be exceeded to avoid oscillatory behavior.

Under the given conditions the coupling stiffness and arm stiffness have only a minor influence on the system oscillatory boundary.
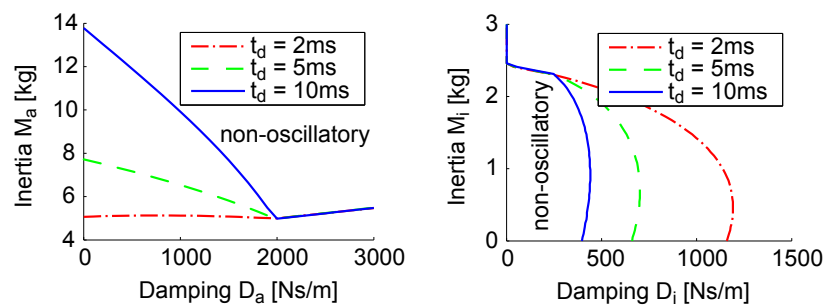

Fig. 3. Simulated system oscillatory boundaries depending on manipulator admittance inertia and damping (left side) and on coupling inertia and damping (right side) for varying time delay. Left side: $M_{i}=1.5 \mathrm{~kg}$, $D_{i}=1000 \mathrm{Ns} / \mathrm{m}, K_{i}=10000 \mathrm{~N} / \mathrm{m}, K_{a}=250 \mathrm{~N} / \mathrm{m}$. Right side: $M_{a}=5.5 \mathrm{~kg}, D_{a}=200 \mathrm{Ns} / \mathrm{m}, K_{a}=250 \mathrm{~N} / \mathrm{m}, K_{i}=10000 \mathrm{~N} / \mathrm{m}$. Both sides: Fixed step sampling time of $1 \mathrm{~ms}$, Euler-Integration (similar to the used robotic system)

The simulations indicate that increasing the arm inertia $M_{a}$ is the primary method in avoiding oscillatory behaviour. The same accounts for the arm damping $D_{a}$ within the range $D_{a}<2 \cdot 10^{3} \frac{\mathrm{Ns}}{\mathrm{m}}$, so this parameter has to be adapted more carefully. The arm spring value $K_{a}$ is not considered as dynamically adapting this value leads to a bad haptic experience for the human even in the static case if the arms are displaced from their desired position. Consequently, it is $\Phi_{c}=\left\{M_{a}, D_{a}\right\}$.

\section{B. FFT-based estimator of $\Theta^{*}$}

Taking user-specific preferences into account how "oscillatory" the current haptic experience during interaction is, one has to approximate the possibly nonlinear human preference $\Theta^{*}$ with a suitable descriptor. For this purpose, an online FFT of the endeffector wrench in combination with a boosting-derived estimator of $\Theta^{*}$ is proposed. This way, oscillations are detected in the frequency domain providing both information about their intensity and frequency.

For the boosting process, four different types of classifiers are used to measure both the current state and the temporal development of the system by considering characteristic properties of multiple frequency spectra.

Let a single discrete spectrum be represented by an indexed set of equidistant frequencies $f_{i}, i=1, \ldots, b$ with corresponding amplitude $A_{i}$. Then the statistical parameters of each spectrum are represented with the mean amplitude $\mu$ and the standard deviation $\sigma$ as

$$
\begin{aligned}
\mu & =\frac{1}{b} \sum_{i=1}^{b} A_{i} \\
\sigma & =\sqrt{\frac{1}{b-1} \sum_{i=1}^{b}\left(A_{i}-\mu\right)^{2}}
\end{aligned}
$$

We define the threshold peak amplitude $A_{p}$ in the spectrum as

$$
A_{p}>\alpha \cdot(\mu+\sigma)
$$

with the adjustable scalar parameter $\alpha$. For taking the temporal development into account, a finite set of the $m_{l}$ respectively $n_{l}$ most recent spectra is considered. Thus the following four different types of classifiers - called weak classifiers - are proposed, see Fig. 4.

- Type 1 classifier detect an oscillation whenever a spectrum contains peaks of a base- and at least $g \geq 1$ harmonic frequencies.

- Type 2 classifier detect an oscillation whenever the same lowest peak frequency is detected at least $m$ times during the last $m_{l}$ timesteps.

- Type 3 classifier detect an oscillation whenever the same peak frequency with the maximum amplitude is detected at least $n$ times during the last $n_{l}$ timesteps.

- Type 4 classifier detect an oscillation whenever the maximum amplitude of the spectrum exceeds a fixed threshold $p$.

The motivation behind weak classifiers of type 1 and 4 is to classify the user-specific preferences by only processing the current spectrum. For type 1 classifiers, even only one harmonic peak frequency is a strong hint for an oscillation. The same accounts for type 4 classifiers and the fixed threshold. Type 2 and 3 weak classifiers are considered if the requirements for type 1 are not fulfilled (missing harmonics) but an oscillation at a certain frequency is present over a longer period of time.

The defined classifiers with varying parameters are combined to strong classifiers employing AdaBoost [13] to represent the possibly nonlinear user-specific preferences.

Since AdaBoost is only capable of learning binary classifiers, multiple datasets have to be used as an input to obtain strong classifiers for different levels of how "oscillatory" the situation feels for the human. To distinguish between $k$ different levels, representative sample data are required for each level to be classified. After applying AdaBoost to all datasets, 

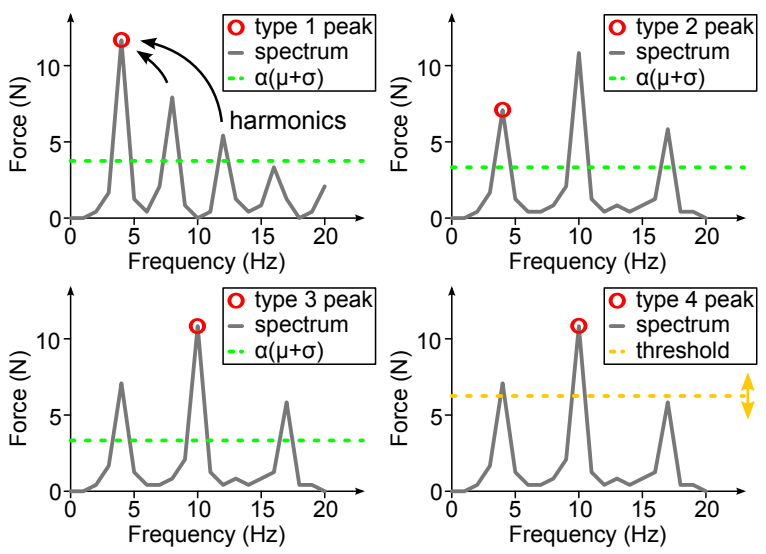

Fig. 4. Visualization of the different classifiers and their functionality.

this results in $k$ strong classifiers $H_{1} \ldots H_{k}=\{0,1\}$ with 0 for a "non-oscillatory" behavior and 1 for an "oscillatory" behaviour of intensity $1, \ldots, k$. As it is not necessarily $H_{i}$ a subset of $H_{i+1}$ due to the human-subjective perception, the sum of all individual classifiers is calculated to obtain the final classifier $\Theta$ according to

$$
\Theta=\sum_{i=1}^{k} H_{i},
$$

as depicted in Fig. 1.

\section{Adaptation strategy}

Given the final classifier $\Theta\left(\Phi_{c}\right)$ and the desired classifier $\Theta_{d}$, let $e$ be the error $\Theta-\Theta_{d}$. Then the loss function is defined similar to [12] as

$$
J\left(\Theta, \Theta_{d}\right)=|e| .
$$

The loss function is usually minimized by a gradient descent approach according to

$$
\frac{d \Phi_{c}}{d t}=-\gamma \frac{\partial e}{\partial \Phi_{c}} \operatorname{sign}(e) .
$$

Under the condition of $\Theta_{d}=0$, i.e. the desired classifier being the one providing the most "pleasant" interaction experience and $\Theta \geq 0$, Eq. 9 can be simplified to

$$
\frac{d \Phi_{c}}{d t}=-\gamma \frac{\partial \Theta}{\partial \Phi_{c}} \text {. }
$$

A problem occurs in calculating $\frac{\delta \Theta}{\delta \Phi_{c}}$, as $\Theta$ consists of discrete values $\Theta=\{0,1, \ldots, k\}$. To resolve this issue, the desired admittance parameters $\Phi_{c, \text { des }}$ and the alteration rate $\frac{d \Phi_{c}}{d t}$ are defined as a discrete function of $\Theta$. These values are obtained through experiments under consideration of the simulation results in III-A and are accessed through a lookup table.

\section{EXPERIMENTAL VALIDATION}

Presented in this section is the application of the method described in Sec. III. To to ensure a proper fitting of the resulting AdaBoost classifier, re- and cross-classification re-

\begin{tabular}{|c|c|c|c|c|c|c|c|}
\hline$H_{1}$. Type & 4 & 4 & 2 & 4 & 1 & 2 & 4 \\
\hline$H_{1}$ : Weight & 2,83 & 2,71 & 1,02 & 0,91 & 0,73 & 0,53 & 0,64 \\
\hline${ }$. Type & 4 & 2 & 4 & 4 & 1 & 3 & 3 \\
\hline $\mathrm{H}_{2}$ : Weight & 0,77 & 0,91 & 0,49 & 0,53 & 0,34 & 0,28 & 0,24 \\
\hline Type & 4 & 1 & 4 & 3 & 1 & 4 & 1 \\
\hline${ }^{3 \cdot}$ Weight & 1,50 & 0,74 & 0,52 & 0,69 & 0,42 & 0,52 & 0,44 \\
\hline $\mathrm{H}_{4}:$ Type & 4 & 1 & $\begin{array}{c}3 \\
056\end{array}$ & $\begin{array}{c}1 \\
043\end{array}$ & $\begin{array}{c}4 \\
060\end{array}$ & $\begin{array}{c}1 \\
034\end{array}$ & 3 \\
\hline
\end{tabular}
sults are presented. Human user studies with ten participants
TABLE I

RESULTING WEAK CLASSIFIER TYPES AND CORRESPONDING WEIGHTS

validate the superiority of the adaptive admittance controller over its non-adaptive counterpart.

\section{A. Classifier boosting}

In order to obtain realistic sample data for the boosting process, a mobile robot with two 7-DoF anthropomorphic arms holding an aluminium bar is used, see Fig. 5. Representative oscillations of different amplitudes were caused and classified by two experienced subjects on a scale from 1 for "low intensity" to 4 for "high intensity". Frequency spectra are obtained through an FFT of the last 512 force data sets at a sampling rate of $1 \mathrm{kHz}$, resulting in spectra of $0-500 \mathrm{~Hz}$ at a frequency resolution of $\frac{1000}{512} \approx 1.95 \mathrm{~Hz}$.

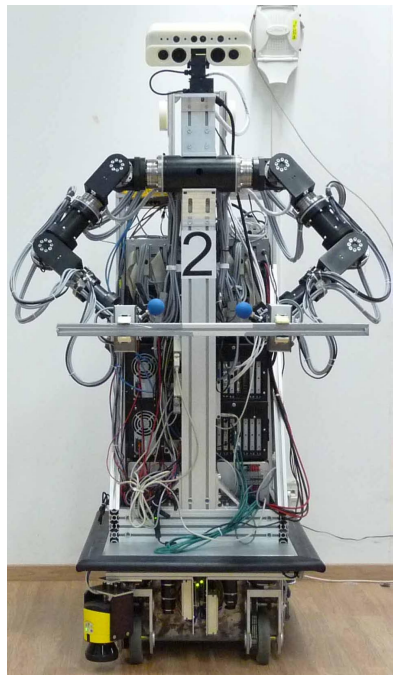

Fig. 5. Experimental setup

Results of the AdaBoost training process over a total of 16200 frequency spectra for the four strong classifiers $H_{1}, \ldots, H_{4}$ are shown in Tab. I. Listed along the vertical axis are the the different strong classifiers, whereas the horizontal axis displays the weight and type of the weak classifiers after 7 iterations of AdaBoost that constitute every strong classifier. As one can see in Tab. I, classifiers of type 4 are assigned the highest weights. Hence it is possible to use only this type for classification to reduce classifier implementation efforts.

In order to evaluate the performance of the AdaBoost trained classifier, re-classification of the same sample set and 
TABLE II

ClassificATION RATES WITH BOOSTED TYPE 1-4 ClASSIFIERS

\begin{tabular}{|c||c|c|}
\hline $\begin{array}{c}\text { Intensity } \\
\text { to classify }\end{array}$ & $\begin{array}{c}\text { Re-classification } \\
\text { rate }\end{array}$ & $\begin{array}{c}\text { Cross-classification } \\
\text { rate }\end{array}$ \\
\hline 1 & $99,99 \%$ & $99,86 \%$ \\
2 & $92,4 \%$ & $87,7 \%$ \\
3 & $97,5 \%$ & $95,3 \%$ \\
4 & $97,8 \%$ & $80,9 \%$ \\
\hline
\end{tabular}

TABLE III

CLASSIFICATION RATES WITH BOOSTED TYPE 4 CLASSIFIERS

\begin{tabular}{|c||c|c|}
\hline $\begin{array}{c}\text { Intrensity } \\
\text { to classify }\end{array}$ & $\begin{array}{c}\text { Re-classification } \\
\text { rate }\end{array}$ & $\begin{array}{c}\text { Cross-classification } \\
\text { rate }\end{array}$ \\
\hline 1 & $99,65 \%$ & $99,62 \%$ \\
2 & $82,3 \%$ & $83,3 \%$ \\
3 & $95,2 \%$ & $94,6 \%$ \\
4 & $87,0 \%$ & $85,4 \%$ \\
\hline
\end{tabular}

cross-classification tests for a new sample set of the same size are performed as shown in Tab. II.

Tab. III provides classification rates using only classifiers of type 4. When being compared to Tab. II in which classifiers of type 1-4 are used, the decrease in the correct classification rate is visible.

\section{B. Adaptive controller evaluation}

For experimental purposes the adaptive controller is tested on the existing admittance controlled setup pictured in Fig. 5. A heuristic static admittance parameterisation of $M_{a}>5.5 \mathrm{~kg}, D_{a}>200 \mathrm{Ns} / \mathrm{m}, K_{a}=250 \mathrm{~N} / \mathrm{m}$ ensures a safe accomplishment of all relevant tasks. The lowest admittance parameters boundary is determined as $M_{a}=3 \mathrm{~kg}, D_{a}=100 \mathrm{Ns} / \mathrm{m}$ and $K_{a}=250 \mathrm{~N} / \mathrm{m}$.

A further decrease of admittance mass and damping allows no safe task accomplishment anymore. The classifierdependent lookup-table for the admittance parameters is defined in Tab. IV.

TABLE IV

LOOK-UP TABLE FOR PARAMETERS ASSIGNMENT

\begin{tabular}{|c||cc|}
\hline \multicolumn{1}{|c||}{$\begin{array}{c}\text { Detected } \\
\text { intensity }\end{array}$} & \multicolumn{2}{c|}{ Desired admittance } \\
$M_{a}[\mathrm{~kg}]$ & $D_{a}[\mathrm{Ns} / \mathrm{m}]$ \\
\hline 0 & 3 & 100 \\
1 & 4 & 120 \\
2 & 5 & 140 \\
3 & 5.5 & 160 \\
4 & 15 & 300 \\
\hline
\end{tabular}

An exemplary sequence of recorded admittance parameters is shown in Fig. 6. Visible are the dynamic, time-dependent detection and classification of oscillations with different intensities and the adaptation of the mass and damping parameters.
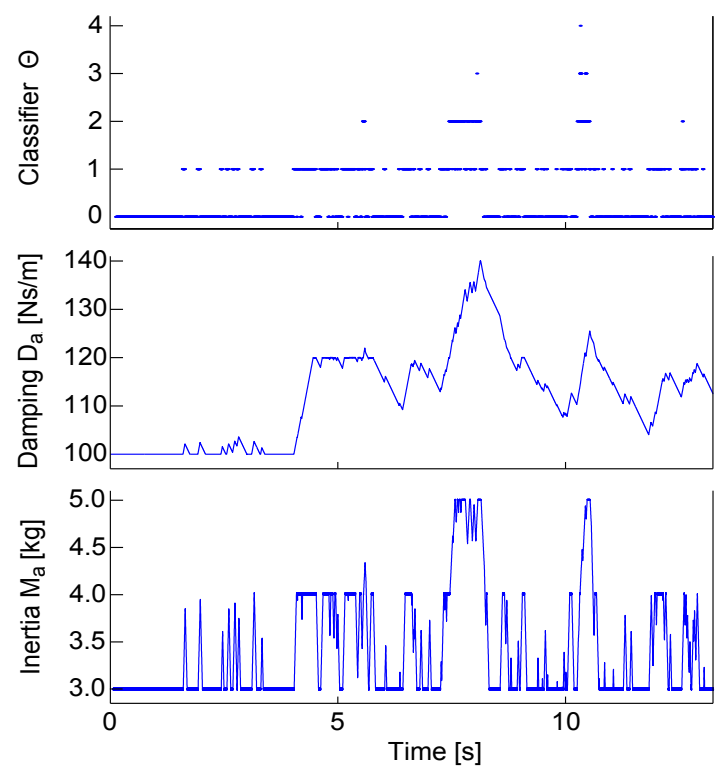

Fig. 6. Adaptive control scheme during a human user study.

In the human user studies the adaptive controller is compared against static controller parameterizations in an experiment with ten participants. Each participant is asked to compare the adaptive controlled setup against four static parameterizations. Three iterations are conducted per participant in which the participants apply either a "light", "medium" or "heavier" contact force. The definition of the contact force levels is left to the subjects. Subsequently the participants rate how "heavy" and "oscillatory" each controller feels on a scale from 1 to 5 . If a statically parameterized, non-adaptive strategy produces oscillatory behaviour causing an abort of the built-in security system of the robot, it is neither rated nor involved in the following iterations due to the guaranteed abort observed in a previous human user study. Strategies involving smaller static admittance parameters are excluded as well in the next iterations for the same reason.

Evaluation results are presented in Tab. V. All individual comparisons for "heavy" and "oscillatory" have been reduced to three cases depending on whether the adaptive controller, none or the static controller feels less "heavy/oscillatory". The column "abort" indicates how often the built-in robot security system shut down for the constant parametrized setup. The adaptive controlled setup never aborts during the experiments.

The goal of the subsequent statistical test is to find out whether there is a reciprocal correlation between "heavy" and "oscillatory" for the two compared controllers, i.e. if controller A is found to be less "heavy", then controller B is found to be less "oscillatory". For this purpose, p-values are evaluated for three null hypotheses

- $H_{0}^{a}$ : There is no difference between a static controller 
TABLE V

RESULTS OF COMPARATIVE EXPERIMENTS

\begin{tabular}{|l||c|c|c|c|}
\hline \multicolumn{5}{|c|}{ Adaptive controller versus static parameterization 1: } \\
\multicolumn{4}{|c|}{$M_{a}=5.5 \mathrm{~kg}, D_{a}=160 \mathrm{Ns} / \mathrm{m}, K_{a}=250 \mathrm{~N} / \mathrm{m}$} \\
\hline Less heavy & $67 \%$ & $33 \%$ & $0 \%$ & \\
Less oscillatory & $7 \%$ & $23 \%$ & $70 \%$ & - \\
p-value $\left(H_{0}^{a}\left|H_{0}^{b}\right| H_{0}^{c}\right)$ & $(0)$ & 0.17 & 0.43 & \\
\hline
\end{tabular}

\begin{tabular}{|l||c|c|c|c|}
\hline \multicolumn{5}{|c|}{ Adaptive controller versus static parameterization 2: } \\
\multicolumn{4}{|c|}{$M_{a}=5 \mathrm{~kg}, D_{a}=140 \mathrm{Ns} / \mathrm{m}, K_{a}=250 \mathrm{~N} / \mathrm{m}$} \\
\hline Less heavy & adaptive & none & static & abort \\
Less oscillatory & $27 \%$ & $53 \%$ & $20 \%$ & - \\
p-value $\left(H_{0}^{a}\left|H_{0}^{b}\right| H_{0}^{c}\right)$ & $20 \%$ & $40 \%$ & $40 \%$ & - \\
\hline
\end{tabular}

\begin{tabular}{|l||c|c|c|c|}
\hline \multicolumn{5}{|c|}{ Adaptive controller versus static parameterization 3: } \\
\multicolumn{4}{|c|}{$M_{a}=4 \mathrm{~kg}, D_{a}=120 \mathrm{Ns} / \mathrm{m}, K_{a}=250 \mathrm{~N} / \mathrm{m}$} \\
\hline Less heavy & adaptive & none & static & abort \\
Less oscillatory & $57 \%$ & $57 \%$ & $10 \%$ & $13 \%$ \\
p-value $\left(H_{0}^{a}\left|H_{0}^{b}\right| H_{0}^{c}\right)$ & $<0.01$ & $17 \%$ & $13 \%$ & \\
\hline
\end{tabular}

\begin{tabular}{|l||c|c|c|c|}
\hline \multicolumn{5}{|c|}{ Adaptive controller versus static parameterization 4: } \\
$M_{a}=3 \mathrm{~kg}, D_{a}=100 \mathrm{Ns} / \mathrm{m}, K_{a}=250 \mathrm{~N} / \mathrm{m}$ \\
\hline Less heavy & adaptive & none & static & abort \\
Less oscillatory & $0 \%$ & $7 \%$ & $0 \%$ & $93 \%$ \\
p-value $\left(H_{0}^{a}\left|H_{0}^{b}\right| H_{0}^{c}\right)$ & $(0)$ & $0 \%$ & $0 \%$ & \\
\hline
\end{tabular}

being less "heavy" and an adaptive controller being less "oscillatory"

- $H_{0}^{b}$ : Both controllers are perceived equally "heavy" and "oscillatory"

- $H_{0}^{c}$ : There is no difference between an adaptive controller being less "heavy" and a static controller being less "oscillatory"

Whereas the p-values for the comparison between the adaptive and static controller with parameterization 1,2 and 4 do not allow a distinctive statement because both controllers were either parameterized too differently or the static controller aborted too often, significant $(\alpha=0.01)$ more persons found the adaptive controller to be less "oscillatory" than the static parameterization 3 to be less "heavy".

\section{DISCUSSION}

The human user studies show that the presented adaptive admittance approach provides a robust way of suiting an admittance controller to human-specific preferences. Whereas the controllers with static parameterization 3 and 4 abort during some/most trials, the adaptive controller works reliable during all experiments.

Parameterization of the slew rate for the dynamic parameter adaptation is a crucial part of the method, compare the rising/falling rates of the Damping $D_{a}$ and Inertia $M_{a}$ in Fig. 6: Whereas a small slew rate is unable to react properly to fast environmental changes causing oscillative behaviour, a large slew rate results in an noticeable oscillating behaviour between different mass and damping parameters. For the experiments, values between $\pm 5-20 \mathrm{~kg} / \mathrm{s}$ for the arm inertia $M_{a}$ and $\pm 50-100 \mathrm{~N} / \mathrm{m}$ for the arm damping $D_{a}$ have proven to work well.

\section{CONCLUSION AND FUTURE WORK}

This paper presents an adaptive admittance control scheme which dynamically varies the admittance parameters to prevent oscillative behaviour of robotic manipulators during human-robot-interaction for tightly-coupled scenarios. A method is proposed to include human preferences into the control design based on a human user study and their evaluation using machine-learning schemes. To model human preferences properly, boosting is proposed for finding a suitable adaptive control scheme. Conducted experiments with ten participants show that the presented approach provides better haptic experience than a fixed-parameter approach.

Ongiong work focuses on the stability proof for the presented adaptive control scheme.

\section{ACKNOWLEDGEMENT}

This work is supported in part within the DFG excellence research cluster Cognition for Technical Systems - CoTeSys (www. cotesys.org).

\section{REFERENCES}

[1] G. The, S. Stramigioli, A. van der Ham, and G. Honderd, "An adaptive admittance or force control for robotic manipulators," Control, pp. 417-421, 1995.

[2] L. Huang, S. S. Ge, and T. H. Lee, "An adaptive impedance control scheme for constrained robots," IJCSS, vol. 5, no. 2, pp. 17-26, 2004

[3] T. Tsumugiwa, R. Yokogawa, and K. Hara, "Variable impedance control based on estimation of human arm stiffness for human-robot cooperative calligraphic task," in ICRA, 2002, pp. 644-650.

[4] S. Kajikawa, "Variable compliance mechanism for human-care robot arm," in IECON, Nov. 2007, pp. $2736-2741$.

[5] C. Mitsantisuk, K. Ohishi, and S. Katsura, "Variable mechanical stiffness control based on human stiffness estimation," in ICM, April 2011, pp. $731-736$.

[6] S. Wolf and G. Hirzinger, "A new variable stiffness design: Matching requirements of the next robot generation," in ICRA, May 2008, pp. $1741-1746$.

[7] R. Ikeura and H. Inooka, "Variable impedance control of a robot for cooperation with a human." in ICRA, 1995, pp. 3097-3102.

[8] R. Ikeura, T. Moriguchi, and K. Mizutani, "Optimal variable impedance control for a robot and its application to lifting an object with a human," in $R O-M A N, 2002$, pp. 500-505.

[9] D. Tsetserukou, R. Tadakuma, H. Kajimoto, N. Kawakami, and S. Tachi, "Intelligent variable joint impedance control and development of a new whole-sensitive anthropomorphic robot arm," in CIRA, 2007, pp. 338-343.

[10] R. Dubey, T. F. Chan, and S. Everett, "Variable damping impedance control of a bilateral telerobotic system," $C S$, vol. 17, no. 1, pp. 37 $-45,1997$.

[11] V. Duchaine and C. M. Gosselin, "General model of human-robot cooperation using a novel velocity based variable impedance control," in WHC, 2007, pp. 446-451.

[12] K. J. Astrom and B. Wittenmark, Adaptive Control, 2nd ed. AddisonWesley Longman Publishing Co., Inc., 1994.

[13] Y. Freund and R. E. Schapire, "A decision-theoretic generalization of on-line learning and an application to boosting," in EuroCOLT, 1995, pp. 23-37. 